\title{
Joint Registration and Segmentation of Dynamic Cardiac Perfusion Images Using MRFs
}

\author{
Dwarikanath Mahapatra and Ying Sun \\ Department of Electrical and Computer Engineering, National University of \\ Singapore, 4 Engineering Drive 3, Singapore 117576 \\ dmahapatra@gmail.com, elesuny@nus.edu.sg
}

\begin{abstract}
In this paper we propose a Markov random field (MRF) based method for joint registration and segmentation of cardiac perfusion images, specifically the left ventricle (LV). MRFs are suitable for discrete labeling problems and the labels are defined as the joint occurrence of displacement vectors (for registration) and segmentation class. The data penalty is a combination of gradient information and mutual dependency of registration and segmentation information. The smoothness cost is a function of the interaction between the defined labels. Thus, the mutual dependency of registration and segmentation is captured in the objective function. Sub-pixel precision in registration and segmentation and a reduction in computation time are achieved by using a multiscale graph cut technique. The LV is first rigidly registered before applying our method. The method was tested on multiple real patient cardiac perfusion datasets having elastic deformations, intensity change, and poor contrast between LV and the myocardium. Compared to MRF based registration and graph cut segmentation, our method shows superior performance by including mutually beneficial registration and segmentation information.
\end{abstract}

\section{Introduction}

Dynamic perfusion magnetic resonance (MR) images are characterized by rapid intensity change over a region of interest, low spatial resolution, poor contrast and noise. Therefore, registration or segmentation of the left ventricle (LV) in cardiac perfusion datasets is a challenging task. However, there are certain characteristics of perfusion images which make it appealing for joint registration and segmentation. The LV is characterized by varying levels of intensity over the image acquisition process. While the changing contrast makes registration difficult, it also helps in segmentation. Clear identification of object boundaries leads to greater accuracy in feature extraction and hence improved registration. Motivated by this scenario and the need to implement a computationally efficient method, we propose a Markov random field (MRF) framework for the joint registration and segmentation of the $\mathrm{LV}$ in cardiac perfusion datasets.

The first work on joint registration and segmentation [1] used an active contour framework to interleave level set segmentation with a feature based registration method. It successfully segmented and registered portal images to CT

T. Jiang et al. (Eds.): MICCAI 2010, Part I, LNCS 6361, pp. 493501, 2010

(C) Springer-Verlag Berlin Heidelberg 2010 
scans. Partial differential equations were used in 2] for joint registration and segmentation while a statistical model was presented in [3]. Wyatt and Noble in 4 combine MRFs and Bayesian estimation for joint registration and segmentation where the use of MRFs is limited to segmentation. There are other methods where registration plays an important role in segmentation and viceversa. In [5] an image is registered to an atlas or a clearly identified object using level sets. Likewise, including shape information in active contours requires a model of shape variation [6] involving accurate registration of training images. In combining registration and segmentation, the major challenges are to ensure convergence and prevent estimates of registration or segmentation parameters adversely affecting each other. Equally important is to define appropriate energy functions and include relevant information for both processes.

The important contribution of our work is in developing an MRF method for the joint registration and segmentation of cardiac perfusion images. Previous methods are based on active contours which, being iterative, have a high computation time, are likely to be trapped in local minima, and are sensitive to initialization. On the other hand discrete optimization techniques for MRFs, like graph cuts, can find a global or strong local optima in less time. We formulate the joint registration and segmentation problem as one of labeling where each label defines the joint occurrence of displacement field (for registration) and segmentation class. The cost function is a combination of the mutual dependency of registration and segmentation information at every label and a multiresolution graph cut optimization reduces the computation time. The rest of the paper is organized as follows: Section 2 gives details about joint registration and segmentation and our MRF formulation. Section 3 presents our experiments and results and we conclude with Section 4

\section{Theory}

\subsection{Joint Registration and Segmentation}

In registration the objective is to match each pixel in the floating image to the most similar pixel in the reference image and the similarity metric depends on the type of images being used. The displacement field is regularized to give a smooth deformation and the smoothness constraints depend upon the registration framework. For B-spline [7] and other curve based registration methods, curve gradients are used as smoothness constraints. In [8], Shekhovstov et al. used MRFs for non-rigid registration where smoothness depends upon the relative displacement between labels. Since the smoothness formulation is not based on the boundary properties of the object being registered it may result in unexpected deformations of the registered image, especially at object boundaries. This shortcoming is overcome by smoothness criteria based on object features which is integral to joint registration and segmentation.

To achieve joint registration and segmentation between a pair of images the following points have to be kept in mind when formulating the energy function: 1) mutual dependency of registration and segmentation is considered; 2) 
registration and segmentation information contribute equally; and 3) estimate of registration parameters does not adversely affect segmentation parameters or vice-versa. The active contour framework in [1] was able to achieve joint registration and segmentation by defining a continuous valued mapping between reference and floating images. A major disadvantage of the active contour framework is the multiple iterations needed for convergence. As with all energy minimization techniques using gradient descent, there is the possibility of the curve being trapped in local minima. The position of the initial curve also influences the final solution. Although this can be overcome by employing a standard segmentation technique to obtain an initial curve, the problems of multiple iterations and getting trapped in local minima still persist. The number of iterations can be greatly reduced by using graph cuts. Graph cuts is based on max flow approach and is very effective in finding the global minima or a strong local minima of discrete MRF energy formulations [9].

\subsection{Markov Random Fields}

MRFs have been previously used with perfusion images for elastic registration [10] and segmentation [11]. Its energy function takes the following form

$$
E(f)=\sum_{s \in P} D_{s}\left(f_{s}\right)+\sum_{(s, t) \in N} V_{s t}\left(f_{s}, f_{t}\right),
$$

where $P$ denotes the set of pixels, $f_{s}$ denotes the label of pixel $s \in P$ in the floating image and $N$ is the set of neighboring pixel pairs. For joint registration and segmentation $f_{s}$ gives both the displacement vector and the segmentation class of pixel $s$, i.e., $f_{s}=\left\{x_{s}^{1}, x_{s}^{2}, L_{s}\right\}$ with $x_{s}=\left\{x_{s}^{1}, x_{s}^{2}\right\}$ denoting displacement along the two axes and $L_{s}$ denoting the segmentation class ( $L_{s}=1$ denotes object and $L_{s}=0$ denotes background). The labels of the entire set of pixels are denoted by $f . D\left(f_{s}\right)=D_{1}\left(x_{s}\right)+D_{2}\left(L_{s}, x_{s}\right)$, is a unary data penalty function derived from observed data and measures how well label $f_{s}$ fits pixel $s$. $V_{s t}$ is a pairwise interaction potential that imposes smoothness and measures the cost of assigning labels $f_{s}$ and $f_{t}$ to neighboring pixels $s$ and $t$. The optimization scheme for (1) using graph cuts is discussed later. Next, we discuss each term of the energy function in detail.

Data Penalty Term: $D_{s}$ assigns a penalty to a pixel $s$ taking on a particular label $f_{s}=\left\{x_{s}^{1}, x_{s}^{2}, L_{s}\right\}$. $D_{s}$ is defined as the sum of two penalty terms. The first term is a function of gradient information and by itself is suitable for registration. The second term includes mutual dependency of registration and segmentation in the penalty term. The following issues are considered in combining the two penalty values: 1) the individual penalties have the same dynamic range, i.e., the difference between their maximum and minimum values should be same; 2) the individual terms are robust for their specific purposes; and 3) the combination of the two terms truly captures the mutual dependency of registration and segmentation. For greater accuracy and robustness a pixel block centered at the pixel is used to calculate the data penalty. We refer to the block centered at pixel $s$ as $s_{b}$. 
Let $I_{f}\left(s_{b}, i\right)$ denote the intensity of the $i$ th pixel of block $s_{b}$ in the floating image $I_{f}$ and $I_{r}\left(s_{b}, i\right)$ denote the corresponding pixel intensity in the reference image $I_{r}$. The pixel block at $s+x_{s}$ is denoted as $s_{b}+x_{s}$ and the intensity of its $i$ th pixel in $I_{r}$ is given by $I_{r}\left(s_{b}+x_{s}, i\right)$. The corresponding edge orientation angles in the two images are given by $\beta_{f}\left(s_{b}, i\right)$ and $\beta_{r}\left(s_{b}+x_{s}, i\right)$ and the corresponding edge magnitudes are given by $M_{f}\left(s_{b}, i\right)$ and $M_{r}\left(s_{b}+x_{s}, i\right)$. Note that the data penalty is for each pixel but is calculated over a pixel block for robustness. $D_{1}\left(x_{s}\right)$ incorporates registration information in the objective function and is a function of gradient orientation information. It is given by

$$
D_{1}\left(x_{s}\right)=\frac{1}{2}\left[1-\frac{\sum_{i} M_{r}\left(s_{b}+x_{s}, i\right) M_{f}\left(s_{b}, i\right) \cos \left(\Delta \beta\left(x_{s}, s_{b}, i\right)\right)}{\sum_{i} M_{r}\left(s_{b}+x_{s}, i\right) M_{f}\left(s_{b}, i\right)}\right],
$$

where $\Delta \beta\left(x_{s}, s_{b}, i\right)=\beta_{r}\left(s_{b}+x_{s}, i\right)-\beta_{f}\left(s_{b}, i\right)$. Edge information has been successfully used to register contrast enhanced images [12] and is a robust feature in the face of intensity changes. $D_{1}$ is a normalized metric that gives values between 0 and 1 with 0 indicating a perfect match. Since $D_{1}$ is a penalty, its value is low for greater similarity between the pixel blocks.

The second penalty term, $D_{2}$ is a function of the mutual dependency of segmentation class and displacement vectors. The LV and myocardium are identified in $I_{f}$ by drawing masks around it and $I_{f}$ is rigidly registered to $I_{r}$. The intensities of pixels inside (outside) the mask are used to create Gaussian models of object (background). Let $p_{f o}(s)$ denote the posterior probability of pixel $I_{f}(s)$ belonging to object and $p_{f b}(s)$ denote its probability of belonging to background. The probability of pixel $I_{r}\left(s+x_{s}\right)$ belonging to object/background is given by $p_{r o}\left(s+x_{s}\right) / p_{r b}\left(s+x_{s}\right)$. Note here that by $s$ we refer to the pixel at $s$. Similarly, $s+x_{s}$ refers to the pixel at location $s+x_{s}$. We do not use $i$ because $D_{2}$ is calculated from individual pixel intensity values. Thus, $D_{2}$ is given by

$$
\begin{aligned}
& D_{2}\left(L_{s}=1, x_{s}\right)=1-\sqrt{p_{f o}(s) \times p_{r o}\left(s+x_{s}\right)} \\
& D_{2}\left(L_{s}=0, x_{s}\right)=1-\sqrt{p_{f b}(s) \times p_{r b}\left(s+x_{s}\right)}
\end{aligned}
$$

For registration and segmentation to jointly influence the penalty term, the segmentation information from the reference and floating image are combined as a function of displacement vectors. If $p_{f o}(s)$ and $p_{r o}\left(s+x_{s}\right)$ both have a value greater than 0.5 the pixel is likely to belong to the object and the penalty for $L_{s}=1$ is low. The corresponding penalty for $L_{s}=0$ is high. If $p_{f b}(s)$ and $p_{r b}\left(s+x_{s}\right)$ have probability values greater than 0.5 , indicating a background pixel, the penalty for background class is low and the corresponding penalty for object class is high. When there is ambiguity over the segmentation class, i.e., $p_{f o}(s) \geq 0.5$ and $p_{r o}\left(s+x_{s}\right)<0.5$ or $p_{f b}(s) \geq 0.5$ and $p_{r b}\left(s+x_{s}\right)<0.5$ then the square root of the product of the probabilities ensures that both the floating and reference image contribute to the penalty of each label.

Pairwise Interaction Term: This term is used to regularize the solution and combines smoothness constraints due to displacement vector and segmentation class. It is defined as 


$$
V_{s t}\left(f_{s}, f_{t}\right)=\lambda_{s t}\left\{\begin{array}{l}
0.002,\left(L_{s}=L_{t}\right) \text { and }\left|x_{s}-x_{t}\right| \leq \sqrt{2} \\
0.002, \quad\left(L_{s} \neq L_{t}\right) \text { and }\left|x_{s}-x_{t}\right| \leq 3 \\
\infty, \\
\text { otherwise. }
\end{array}\right.
$$

$\lambda_{s t}$ is a spatially varying weight that depends upon the intensity difference between the neighboring pixels of the floating image and is given by

$$
\lambda_{s t}=\exp \left\{-\left[I_{f}(s)-I_{f}(t)\right]^{2}\right\} .
$$

When neighboring pixels have the same segmentation class $\left(L_{s}=L_{t}\right)$, they are likely to have similar displacements because pixels on the same object tend to move together. Thus we constrain the maximum relative displacement between the pixels to be $\sqrt{2}$ pixels. If neighboring pixels have different segmentation class $\left(L_{s} \neq L_{t}\right)$, then they can have different displacements since pixels on different objects may move differently. Therefore we allow relative displacement between such pixels to be up to 3 pixels.

Optimization using Graph Cuts: The energy function is optimized using graph cuts [9] which is suitable for discrete MRF labeling problems. Its ability to enforce piecewise coherence makes it especially suitable for vision applications. We represent pixels as nodes $V_{p}$ in a graph $G$ which also consists of a set of directed edges $E$ that connect nodes. For $l$ labels, $l$ terminal nodes are created. First, \pm 6 displacement positions along $x$ and $y$ axis are defined with a step of 1 pixel between two consecutive positions. For every position there are two segmentation classes. The total number of labels are $2 \times(2 \times 6+1)^{2}=338$. In the second stage \pm 4 displacements along each axis with a step of 0.5 pixel is defined and the total number of labels is 162 . Pixel blocks of size $5 \times 5$ were used for determining the penalty values.

\section{Experiments and Results}

Registration Results: Cardiac images were acquired on Siemens Sonata MR scanners following bolus injection of Gd-DTPA contrast agent. The pixel spacing ranges from $(1.5 \times 1.5)-(2.8 \times 2.8) \mathrm{mm}^{2}$. Contrast agent flows into the right ventricle $(\mathrm{RV})$, then into the LV and finally into the myocardium. The acquired datasets were all in 2D and a total of 10 datasets were used to test our method. Each dataset had 60 frames. In some of the datasets, the images before contrast enhancement did not show the LV and were discarded. The total number of test images were 538 with one reference image from each dataset. The LV nad myocardium was identified in each image by drawing masks and then rigidly registered before applying our method. We compared the registration performance of our method $(J R S)$ and an MRF method that does not use segmentation information, i.e.,

$$
E(f)=\sum_{s \in P} D_{1}\left(f_{s}\right)+\sum_{(s, t) \in N} V_{s t}\left(f_{s}, f_{t}\right),
$$


where $f_{s}=x_{s}=\left\{x_{s}^{1}, x_{s}^{2}\right\}$ and $V_{s t}$ is given by

$$
V_{s t}\left(f_{s}, f_{t}\right)=\left\{\begin{array}{lr}
0.002,\left|f_{s}-f_{t}\right| \leq \sqrt{2} \\
\infty, & \text { otherwise }
\end{array}\right.
$$

The reference image (Fig. 1 (a)) and floating image (Fig. 1 (b)) pair was chosen such that there is intensity change between them as well as noticeable deformations. The deformations are highlighted by the difference image in Fig. 1] (c). Dark areas in the difference image are the ones with negative intensity difference and bright areas correspond to positive intensity difference values. Areas in grey have zero intensity difference. The difference image after registration using only MRFs (Fig. 1 (d)), does not register all the deformations especially at the boundaries of the RV. The difference image obtained after registration using $J R S$ (Fig. 1 (e)) shows noticeable improvement in registration performance and inclusion of segmentation information plays a crucial role in it.

The epicardium and endocardium were manually identified in the reference image and all floating images. From the obtained deformation field we register the LV and myocardium in the floating image and calculate the error between the registered contours and those in the reference image. The measured error is the average distance between each point on the registered contour and the nearest point on the reference contour. We also show the outline of the registered contours (in red) on the reference image using JRS (Fig. 1 (f)) and MRFs (Fig. 1 (g)). The contours are better registered using $J R S$ as is evident from the distance between the registered epicardium and endocardium. The quantitative error measures before and after registration using $J R S$ and MRFs are shown in Table 1. While the average registration error after using MRFs is greater than $1 \mathrm{~mm}$, in case of $J R S$ it is well below $1 \mathrm{~mm}$. In fact the maximum registration error for $J R S$ hardly goes above $1 \mathrm{~mm}$, with only a few datasets exhibitng that.

The deformed grids obtained from MRFs and $J R S$ are shown, respectively, in Figs. 1 (h) and (i). The deformation field obtained from MRFs and $J R S$ are smooth. Even though segmentation information has been incorporated into the cost function, the smoothness constraint has been appropriately formulated to avoid folding. The registration errors given in Table 1 show the greater accuracy obtained for $J R S$. While MRFs, without any segmentation information, have higher registration error, they do not result in folding of deformation field. The relative displacement threshold in $V_{s t}(\sqrt{2}$ in our case) plays an important role in influencing the smoothness of the deformation field. An increase in threshold leads to a disparate displacement field, i.e., neighboring pixels do not have coherent motion. To avoid such issues, the relative displacement between neighboring pixels is constrained to be within $\sqrt{2}$ pixels, which is the maximum distance between neighbors. Such an arrangement is necessary for discrete valued cost functions.

Segmentation Results: The LV and myocardium in each floating image are also separately segmented using graph cuts [1]. Figures 2 (a) and (b) show respectively the outline of segmented mask using graph cuts and $J R S$ on the same floating image. By segmentation results using $J R S$ we refer to the segmentation 


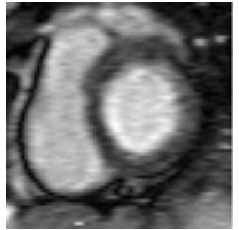

(a)

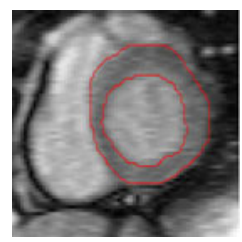

(f)

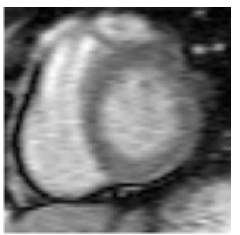

(b)

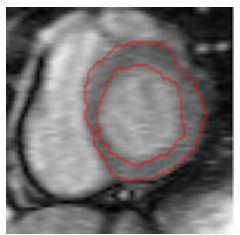

(g)

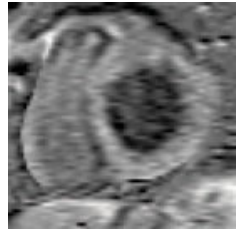

(c)

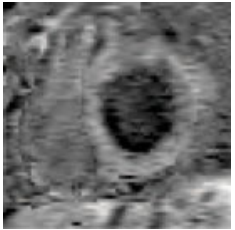

(d)

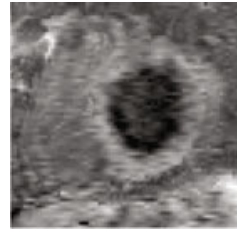

(e)

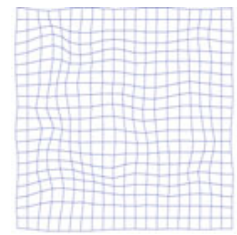

(i)

Fig. 1. Registration results for cardiac perfusion images. (a) Reference image; (b) floating image; difference image (c) before registration; (d) after registration using MRFs and (e) after registration using $J R S$. Superimposed outline of registered contours using (f) $J R S$ and (g) MRFs. Deformed grids obtained from (h) JRS and (i) MRFs.

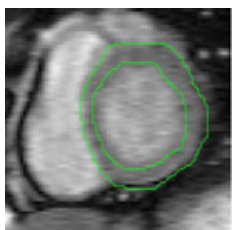

(a)

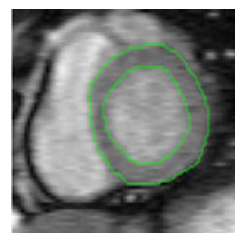

(b)

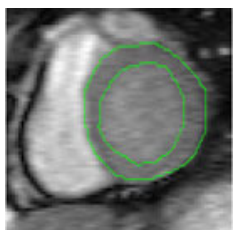

(c)

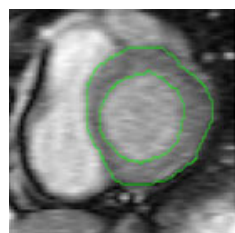

(d)

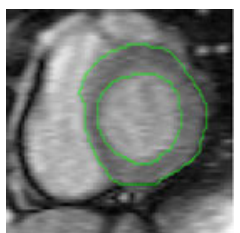

(e)

Fig. 2. Segmentation results for cardiac perfusion images. Outline of segmented mask in green using (a) graph cuts; and (b) JRS; (c)-(e) show the outline of the deformed mask in three floating images. In each floating image, the deformed mask was obtained by deforming the segmented mask of the reference image according to the corresponding displacement field.

class from the labels of each pixel in the floating image. $J R S$ results in greater segmentation accuracy than graph cuts for images with poor contrast between LV and myocardium. The segmented mask from the reference image is deformed using the deformation field of different frames of the sequence. This deformed mask is then superimposed on the corresponding floating image as shown in Figs. 2 (c)-(e). These results show that registration and segmentation mutually benefit each other, i.e., the epicardium and endocardium are not only segmented accurately but also registered to the reference image. Table 1 also shows the different error measures for segmentation. The average Dice Metric $(D M)$ and Root Mean Square (RMS) values for each dataset are shown in Fig. 3. JRS consistently shows higher $D M$ and lower $R M S$ values than graph cuts. The average RMS value for each dataset is close to 1 pixel and the average $D M$ is above $90 \%$ 


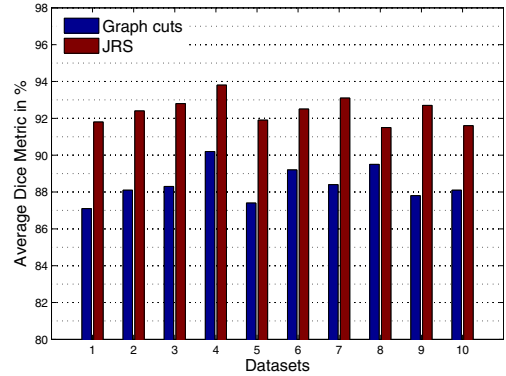

(a) Dice Metric values

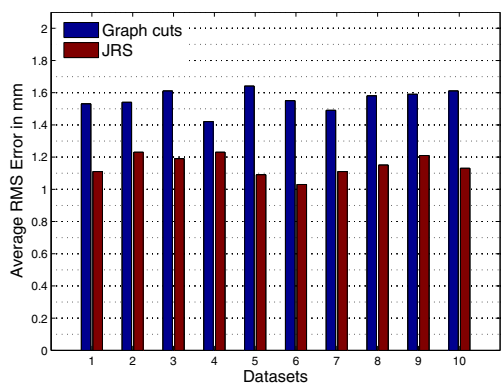

(b) RMS values

Fig. 3. Quantitative segmentation results for 10 datasets. (a) average $D M$ values; (b) average RMS value. Brown bars show results for $J R S$ and blue bars show results for graph cuts.

Table 1. Summary of registration and segmentation performance on cardiac perfusion datasets. The values indicate average and standard deviations for all datasets.

\begin{tabular}{|l|l|l|l||l|l|l|l|}
\hline \multirow{2}{*}{} & \multicolumn{3}{|c|}{ Registration Error (mm) } & \multicolumn{3}{c|}{ Segmentation Result } \\
\cline { 2 - 8 } & $\begin{array}{l}\text { Before } \\
\text { Registration }\end{array}$ & After Registration & \multicolumn{2}{|c|}{ Graph Cuts } & \multicolumn{2}{c|}{$J R S$} \\
\cline { 2 - 8 } & MRFs & JRS & $R M S(\mathrm{~mm})$ & $D M(\%)$ & $R M S(\mathrm{~mm})$ & $D M(\%)$ \\
\hline Epicardium & $2.2 \pm 1.2$ & $1.0 \pm 0.2$ & $0.7 \pm 0.4$ & $1.45 \pm 0.43$ & $88.6 \pm 1.6$ & $1.13 \pm 0.32$ & $92.1 \pm 1.1$ \\
\hline Endocardium & $2.8 \pm 1.0$ & $1.1 \pm 0.3$ & $0.6 \pm 0.3$ & $1.65 \pm 0.31$ & $89.2 \pm 0.9$ & $1.11 \pm 0.39$ & $92.7 \pm 0.8$ \\
\hline Overall & $2.6 \pm 1.1$ & $1.1 \pm 0.2$ & $0.5 \pm 0.2$ & $1.52 \pm 0.34$ & $88.93 \pm 1.2$ & $1.11 \pm 0.34$ & $92.5 \pm 0.9$ \\
\hline
\end{tabular}

(values above $80 \%$ indicate excellent agreement with manual segmentation). For graph cuts $D M$ values less than $90 \%$ are attributed mainly to the poor contrast between LV and myocardium in many images.

\section{Conclusion}

We have proposed a novel MRF based method for the joint segmentation and non-linear registration of the LV in perfusion cardiac images. Our method is different from previous works using active contours, and MRFs ensure less computation time and high accuracy. The problem was formulated as one of finding the appropriate labels. The labels give the displacement vector and segmentation class for each pixel. The cost function depends on contrast invariant edge information, segmentation class, and mutual dependency of registration and segmentation. The final labels are obtained by minimizing the cost function in a multiresolution graph cut implementation. The coarse to fine graph cut implementation gives sub-pixel accuracy for registration and segmentation. The performance of our method was compared with an MRF based registration method using only gradient information and a graph cut based segmentation method. Quantitative and visual results are shown for the registration of the epicardium and endocardium. For comparing segmentation performance, Dice Metric and 
RMS errors between segmented LV and reference manual segmentation are calculated. All sets of results show the superior performance of our method compared to separate registration and segmentation method. In future work we aim to extend our method for 3D datasets and other imaging modalities, and also optimize it for less computation time.

Acknowledgment. The authors thank Siemens Corporate Research, NJ, USA, for providing the datasets, and acknowledge the support by NUS grant R-263000-470-112.

\section{References}

1. Yezzi, A., Zollei, L., Kapur, T.: A variational framework for joint segmentation and registration. In: Proc. MMBIA, pp. 44-51 (2001)

2. An, J.-h., Chen, Y., Huang, F., Wilson, D., Geiser, E.: A variational PDE based level set method for a simultaneous segmentation and non-rigid registration. In: Duncan, J.S., Gerig, G. (eds.) MICCAI 2005. LNCS, vol. 3749, pp. 286-293. Springer, Heidelberg (2005)

3. Flach, B., Sara, R.: Joint non-rigid motion estimation and segmentation. In: Klette, R., Žunić, J. (eds.) IWCIA 2004. LNCS, vol. 3322, pp. 631-638. Springer, Heidelberg (2004)

4. Wyatt, P.P., Noble, J.A.: MAP MRF joint segmentation and registration. In: Dohi, T., Kikinis, R. (eds.) MICCAI 2002, Part I. LNCS, vol. 2488, pp. 580-587. Springer, Heidelberg (2002)

5. Tsai, A., Yezzi, A., Wells, W., Tempany, C., Tucker, D., Fan, A., Grimson, W., Willsky, A.: A shape-based approach to the segmentation of medical imagery using level sets. IEEE Trans. Med. Imag. 22(2), 137-154 (2003)

6. Cootes, T., Beeston, C., Edwards, G., Taylor, C.: Unified framework for atlas matching using active appearance models. In: Kuba, A., Sámal, M., ToddPokropek, A. (eds.) IPMI 1999. LNCS, vol. 1613, pp. 322-333. Springer, Heidelberg (1999)

7. Rueckert, D., Sonoda, L.I., Hayes, C., Hill, D.L.G., Leach, M.O., Hawkes, D.J.: Nonrigid registration using free-form deformations: application to breast MR images. IEEE Trans. Med. Imag. 18, 712-721 (1999)

8. Shekhovtsov, A., Kovtun, I., Hlavác, V.: Efficient MRF deformation model for nonrigid image matching. Comput. Vision Image Understand. 112(1), 91-99 (2008)

9. Boykov, Y., Veksler, O.: Fast approximate energy minimization via graph cuts. IEEE Trans. Pattern Anal. Mach. Intell. 23, 1222-1239 (2001)

10. Mahapatra, D., Sun, Y.: Nonrigid registration of dynamic renal MR images using a saliency based MRF model. In: Metaxas, D., Axel, L., Fichtinger, G., Székely, G. (eds.) MICCAI 2008, Part I. LNCS, vol. 5241, pp. 771-779. Springer, Heidelberg (2008)

11. Boykov, Y., Funka-Lea, G.: Graph cuts and efficient N-D image segmentation. Intl. J. Comp. Vis. 70(2), 109-131 (2006)

12. Sun, Y., Jolly, M.P., Moura, J.M.: Contrast-invariant registration of cardiac and renal MR perfusion images. In: Barillot, C., Haynor, D.R., Hellier, P. (eds.) MICCAI 2004. LNCS, vol. 3216, pp. 903-910. Springer, Heidelberg (2004) 